\title{
ESCLEROSE LATERAL AMIOTRÓFICA
}

\author{
VARIANTE DISTAL DOS MEMBROS INFERIORES
}

\author{
APRESENTAÇÃO DE TRÊS CASOS
}

\author{
J. M. GODOY * - M. SKACEL ** - S. L. BALASSIANO *** - J. R. M. NEVES *** \\ G. S. GOFFREDO FILHO **** - R. B. DE CASTRO **** \\ D. H. NICARETTA ***** - R. G. S. SOUZA ***** - M. H. F. MENDES *****
}

RESUMO - Os autores apresentam três casos de pacientes com esclerose lateral amiotrófica, variante de Patrikios, chamando a atenção para a importância do diagnóstico diferencial desta com as neuropatias periféricas.

Amyotrophic lateral sclerosis: Patrikios form. Report of three cases.

SUMMARY - The, authors describe the neurological findings in ALS, Patrikios form, of three patients, calling attention for the differential diagnosis with peripheral neuropathies.

A esclerose lateral amiotrófica (ELA) é doença de causa desconhecida ainda nos dias de hoje, apesar dos conhecimentos sobre vários de ccus aspectos terem avançado desde a época de Charcot. Numerosas teorias foram propostas para sua patogênese, porém nenhuma até o momento mostrou-se unificadora. Estas teriam que explicar os diferentes graus de incidência em diversas áreas geográficas, as formas de apresentação clínica distintas, tempo de evolução, bem como a vulnerabilidade e a resistência de determinadas populações neuronais. É possível que estejamos diante de várias entidades anátomo-clínicas de etiologias múltiplas e que se apresentam de forma semelhante. Rowland ${ }^{18}$ define bem nosso estágio atual de conhecimento quando afirma que «qualquer notificação de melhora nos pacientes com esta trágica doença merece avaliação cuidadosa. Todas ou nenhuma das teorias sobre a patogênese podem ser válidas; nenhuma, contudo, poderá ser afastada sem que haja uma pesquisa profunda». Empregamos o termo ELA quando o paciente apresenta manifestações clínicas secundárias à lesão dos primeiro e segundo neurônios motores. Diversas variantes clínicas são conhecidas e Bondouelle ${ }^{3}$ as subdivide conforme se segue: (a) forma clássica $(56 \%)$, que se inicia nos membros superiores e, posteriormente, evolui para os inferiores e núcleos motores bulbares; (b) forma bulbar (28\%), na qual disfagia, disartria e atrofia com miofasciculações da língua constituem as manifestações iniciais, seguindo-se o acometimento dos membros superiores e inferiores; (c) forma dos membros inferiores $(16 \%)$ que, segundo Patrikios ${ }^{14}$, pode se apresentar em duas variantes — flácida ou pseudo-políneurítica e espástica.

Neste estudo é analisado o quadro clínico de três pacientes com ELA distai dos membros inferiores, chamando-se a atenção para a importância do diagnóstico diferencial com as neuropatías periféricas (NP).

* Professor Adiunto de Neurologia da Universidade Estadual do Rio de Janeiro (UERJ); ** Professor Auxiliar de, Neurologia da UERJ; *** Professor Assistente de Neurologia da UERJ; ***** Interno e ***** Residente do Hospital Universitário Pedro Ernesto (HUPE-UERJ).

Dr. José Mauricio Godoy - Hospital Universitário Pedro Ernesto - Av. 28 de Setembro 77 $2^{\circ}$ andar - 20551 Rio de Janeiro RJ - Brasil. 


\section{CASUÍSTICA}

Foram estudados três pacientes internados no Serviço de Neurologia do HUPE-UERJ: no 1 (registro 770293), no 2 (registro 796102) e no 3 (registro 794357). Todos eram do sexo masculino e da raça branca e a idade de início da doença oscilava entre 45 e 68 anos. Inexistiam antecedentes familiares e exposição profissional a metais pesados. Os três enfermos apresentaram como queixa inicial, fraqueza distai do(s) membro(s) inferior(es) e fenômenos sensitivos subietivos (cãibras e parestesias) no mesmo território. O exame clínico era normal e o neurológico acusava pé equino bilateral nos pacientes no 1 e no 3 e à direita no de no 2 (Fig. 1), com consequente marcha escarvante. As funções superiores, nervos cranianos e as sensibilidades não mostravam alterações. Todos os enfermos apresentavam miofasciculações nos quatro membros, sendo que no no 2 estas eram também visíveis na musculatura do tórax e abdomem. Havia nítida redução da força muscular com atrofia dos flexores dorsais dos pês nos pacientes no 1 e no 3, mesmo déficit observado à direita no no 2, acrescido de diminuição da força e atrofia do «Quadriceps femoris» homolateral (Fig. 1).

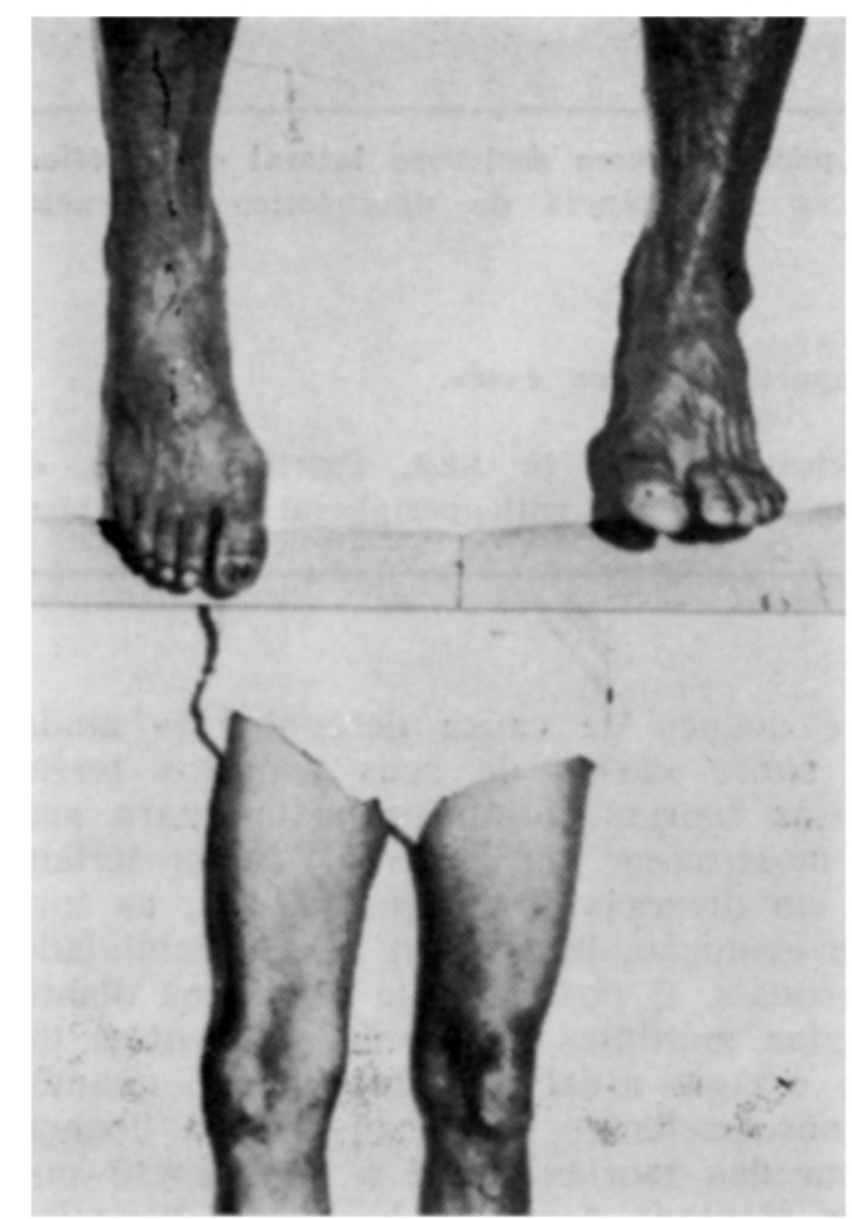

Fig. 1 - Aspectos do paciente 2. Em cima, pe equino à direita. Em baixo, atrofia da coxa à direita.

Os reflexos osteotendinosos estavam exaltados nos quatro membros com acinesia dos Aquileus nos três enfermos. Havia sinal de Babinski no no 1 e no 3 , este último com sinal de Hoffmann bilateral. Os cutâneo-plantares eram indiferentes no no 2 . Na tabela 1 são resumidos os achados do exiame neurológico nos três pacientes.

\begin{tabular}{ccccccccc}
\hline Paciente & Fraq. Atr. & A.S.S. & R.A. & Babinski & Hoffmann & Mf. & BTPEx. & A.o.S. \\
\hline \hline 1 & $(+)$ & $(+)$ & $(-)$ & $(+)$ & $(-)$ & $(+)$ & $(+)$ & $(-)$ \\
2 & $(+)$ & $(+)$ & $(-)$ & $(-)$ & $(-)$ & $(+)$ & $(+)$ & $(-)$ \\
3 & $(+)$ & $(+)$ & $(-)$ & $(+)$ & $(+)$ & $(+)$ & $(+)$ & $(-)$ \\
\hline
\end{tabular}

Trabela 1 - Achados do exame neurologico.

(-) ausente; (+) presente; Iraq. Atr., fraqueza e atrofia distal nos membros inferiores; A.S.S., alteraçes subjetivas da sensibilidade; R.A., reflexo aquileu; Mf., miofasciculacóses; BTPEx., reflexos bicipitais, tricipitais e patelares exaltados; A.O.S., alteraçes objetivas da sensibilidade. 
As análises séricas (hemograma, velocidade de hemossedimentação, glicose, uréia, creatinina, proteínas totais e frações, imuneletroforese das proteínas, cálcio, fósforo, fosfatase alcalina e creatinofosfoquinase), bem como o estudo do líquido cefalorraquidiano (LCR) com prova de Stookey foram normais. Não foi realizada imuneletroforese das proteínas do LCR $\mathrm{O}$ estudo radiográfico da coluna vertebral foi normal nos enfermos $\mathrm{nU}$ e $\mathrm{n} \ll 2$, tendo evidenciado anormalidades degenerativias no n? 3. Mielografia foi indicada neste último, que se mostrou normal. As velocidades de condução motora e sensitiva estavam reduzidas (Fig. 2) e a eletromiografia (EMG) com agulha concêntrica demonstrou presença de fibrilaçôes e fasciculações (Fig. 3) em todos os pacientes, não somente nos grupos musculares dos apêndices superiores como também dos inferiores. Levando-se em conta os achados clínicos e eletrofisiológicos, ficou estabelecido o diagnóstico de ELA, variante de Patrikios, em todos os enfermos.

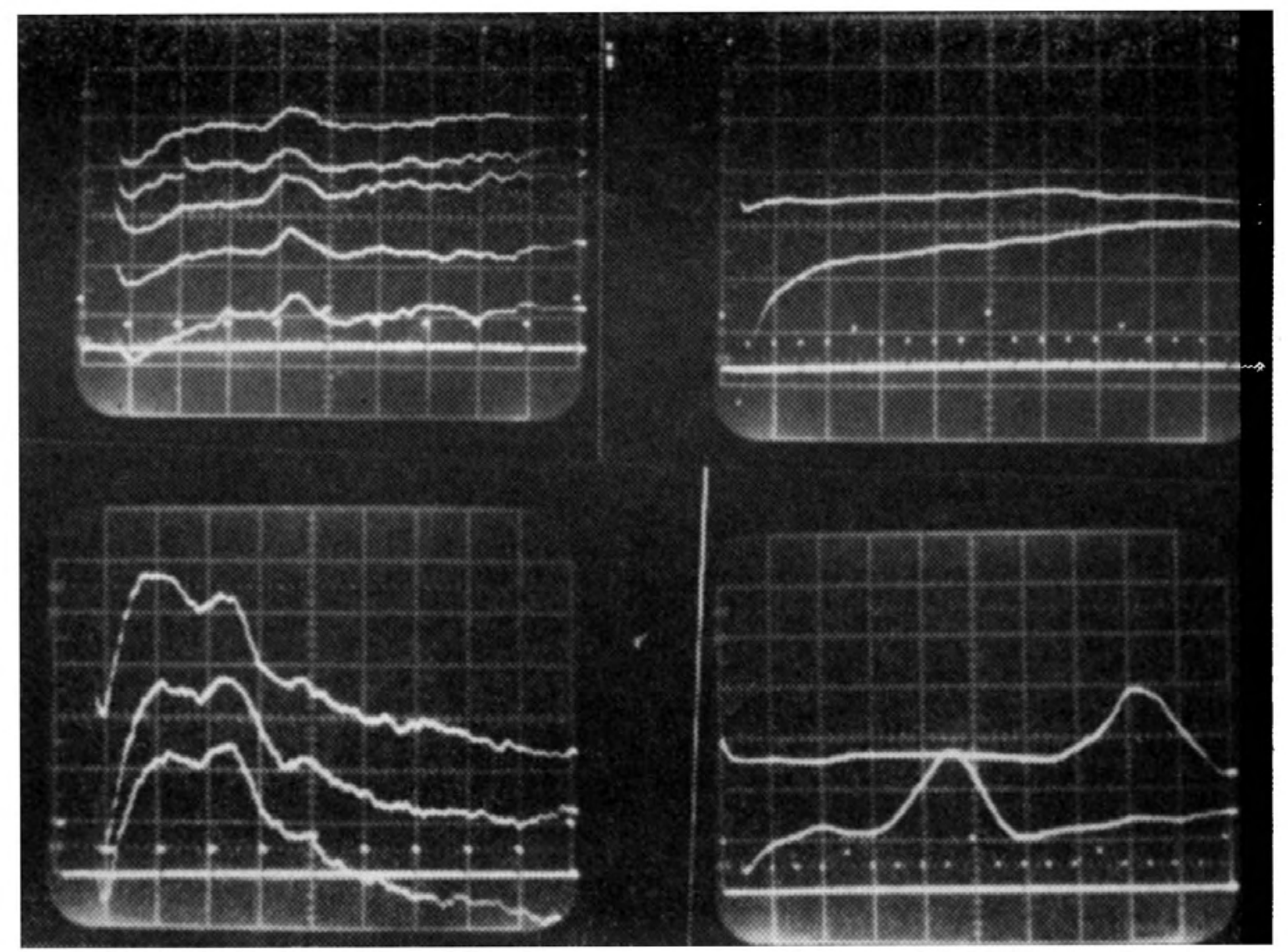

Fig. 2 - VCN. A esquerda VCN do nervo sural (pacientes 1 e 3). A direita, VCN do nervo peroneal (pacientes 1 e 3).

\section{COMENTÁRIOS}

A ELA caracteriza-se pela presença de manifestações clínicas advindas de lesão dos motoneurônios da medula espinhal e do tronco cerebral, acrescida de sinais e sintomas piramidais, todos em níveis variáveis. Sua etiologia permanece obscura, porém, alguns autores postulam a participação de múltiplos fatores na gênese da doença $8,13,15,19,21$.

A similitude clínica entre certas formas de ELA e NP foi, inicialmente, descrita por Patrikios ${ }^{14}$. Dyck ${ }^{7}$, em 1982, inicia sua exposição da forma seguinte: «São as neuropatías periféricas e a doença do neurônio motor separáveis? Eu responderia que algumas são e muitas não. A separação rígida entre as degenerações neuronal (ELA) e axonal (NP) é, frequentemente, difícil».

De todos os itens implicados na fisiopatogenia da ELA, o tóxico e o imunológico são sabidamente causadores de NP, em especial a saturnínica' e a secundária às gamapatias monoclonais ${ }^{12}$. Considerando as síndromes paraneoplásicas como fenômeno imunológico, alguns autore $s^{2} »^{10}$ fizeram alusão acerca da ELA como integrante, assim como as NP, deste grupo. Entretanto, a associação de neoplasias com ELA parece ser meramente casual. Dentre os diversos fatores, possivelmente, ligados à patogenia da ELA, o tóxico foi postulado por Aran', quando da descrição de 11 enfermos com doença do neurônio motor, dos quais três apresentavam história pre- 


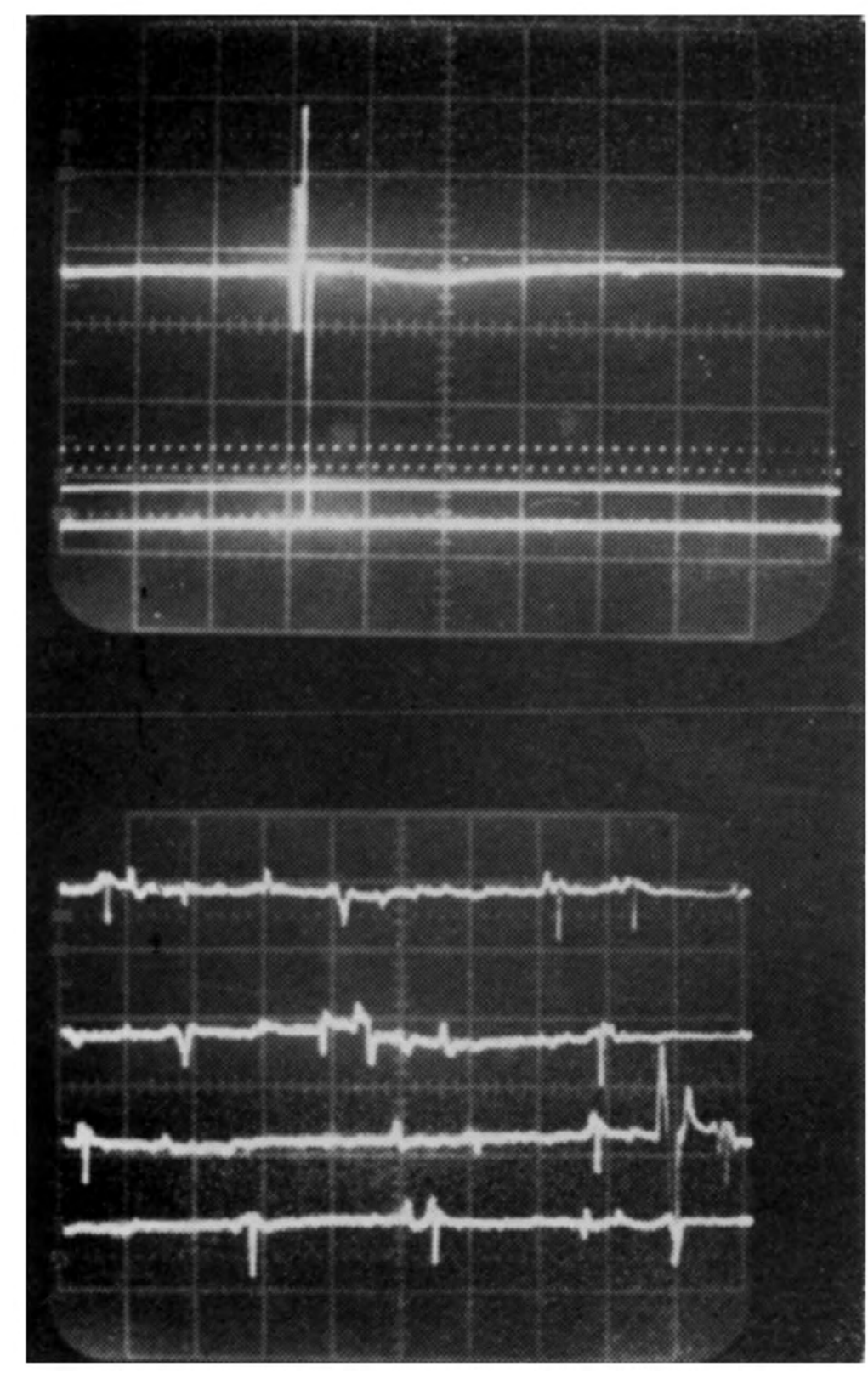

Fig. 3 - Fasciculação (em cima) e fibrilações (em baixo): pacientes 1 e 3 .

gressa de exposição ao chumbo. Posteriormente, outros autores ${ }^{4} »^{5} \cdot{ }^{20} »^{22}$ relataram sobre a associação da ELA com metais pesados. Por outro lado, a possível ligação entre a doença e disproteinemias ${ }^{11}$, estimulou neurologistas dos quatro cantos do mundo. Contudo, os achados não foram suficientes para afastar apenas a relação fortuita das duas. Apesar das alterações imunológicas observadas precocemente na ELA ${ }^{16}$, a importância de tal fenômeno permanece um enigma.

A variante ora apresentada constitui grande «armadilha» para os médicos, mesmo para aqueles que exercem especialidade ligada às neurociências. Nossa experiência demonstra que enfermos com o diagnóstico final de ELA são, inicialmente, encaminhados aos ambulatórios de medicina interna, dermatologia sanitária ou ortopedia com a hipótese de NP a esclarecer. Levando-se em conta que alguns enfermos iniciam o quadro clínico com manifestações consequentes à lesão do segundo neurônio motor e fenômenos sensitivos subjetivos, e que as miofasciculações raramente são referidas por eles ${ }^{17}$, tal equívoco torna-se aceitável para o não especialista. Todavia, não existe NP cursando com hiperreflexia osteotendinosa ou outros sinais de liberação piramidal. Além disso, alterações objetivas da sensibilidade, comuns nas NP, inexistem na ELA esporádica.

Embora as anormalidades eletrofisiológicas mais importantes na ELA sejam observadas por EMG com agulha concêntrica, a análise das velocidades de condução motora e sensitiva (VCN) constitui dado fundamental nesses enfermos. Enquanto esta mostra-se, de forma acentuada, reduzida nas NP, na ELA as alterações são discretas. A VON nas fibras de condução mais rápida pode estar ligeiramente lentificada e a latência distai prolongada nos portadores de ELA ${ }^{6}$. Assim sendo, o eletromiogra- 
fista deverá estar atento durante a realização do exame; caso contrário poderá induzir ao diagnóstico equivocado de NP.

A importância em lembrar a existência desta entidade visa chamar a atenção para a extrema variabilidade clínica da ELA que, nos três pacientes apresentados neste artigo, simula lesão do nervo periférico. $O$ avanço tecnológico no campo da neurologia faz com que, muitas vezes, o médico se afaste das técnicas propedêuticas indispensáveis para o correto diagnóstico dos enfermos.

fista deverá estar atento durante a realização do exame; caso contrário poderá induzir ao diagnóstico equivocado de NP.

A importância em lembrar a existência desta entidade visa chamar a atenção para a extrema variabilidade clínica da ELA que, nos três pacientes apresentados neste artigo, simula lesão do nervo periférico. O avanço tecnológico no campo da neurologia faz com que, muitas vezes, o médico se afaste das técnicas propedêuticas indispensáveis para o correto diagnóstico dos enfermos.

\section{REFERÊNCIAS}

1. Aran FA. Recherches sur une maladie non encore décrite du système musculaire (atrophie musculaire progressive). Arch Gén Méd 1850, 24 : 4.

2. Barron KD, Rodichok LB. Cancer and disorders of motor neurons. In Rowland LP (ed): Human Motor Neuron Diseases. New York: Raven Press, 1982, p 267.

3. Bonduelle M. Amyotrophic lateral sclerosis. In Vinken PJ, Bruyn GW (eds): Handbook of Clinical Neurology. Amsterdam: North-Holland, 1975, p 281.

4. Campbell AMG, Williams ER, Barltrop D. Motor neuron disease and exposure to lead. J Neurol Neurossurg Psychiat 1970, $33: 877$.

5. Conradi S, Ronnevi L-O, Norris FH. Motor neuron disease and toxic metals. In Rowland LP (ed): Human Motor Neuron Diseases. New York: Raven Press, 1982, p 201.

6. Daube JR. Electrophysiologic studies in the diagnosis and prognosis of motor neuron disease. Neurol Clin 1985, 3:473.

7. Dyck PJ. Are motor neuropathies and motor neuron diseases separable? In Rowland LP (ed): Human Motor Neuron Diseases. New York: Raven Press, 1982, p 105.

8. Godoy JM. Alterações no metabolismo do cálcio e fósforo, na doença do neurônio motor. Tese apresentada para a obtenção do grau de Mestre em Neurologia. Universidade Federal do Rio de Janeiro, 1989.

9. Goldstein NP, Mc Call JT, Dyck PJ. Metal neuropathy. In Dyck PJ, Thomas PK, Lambert EH (eds): Peripheral Neuropathy. Philadelphia: Saunders, 1975, p 1227.

10. Henson RA, Urich H. Miscellaneous, and some possibly fortuitous associations. In Henson RA, Urich H (eds): Cancer and the Nervous System: The Neurological Manifestations of Systemic Malignant Disease. Oxford: Blackwell, 1982, p 432.

11. Latov N. Plasma cell dyscrasia and motor neuron disease. In Rowland LP (ed): Human Motor Neuron Diseases. New York: Raven Press, 1982, p 273.

12. Mc Leod JG, Wlash JC. Neuropathies associated with paraproteinemias and dysproteine-ᄀ mias. In Dyck PJ, Thomas PK, Lambert EH (eds): Peripheral Neuropathy. Philadelphia: Saunders, 1975, p 1012.

13. Müller WK, Hilgenstock F. An uncommon case of amyotrophic lateral sclerosis with isolation of a virus from the CSF. J Neurol 1975, $211: 11$.

14. Patrikios J. Contribution à l'étude des formes cliniques et de l'anatomie pathologique de la sclérose latérale amyotrophique. Thesis. Paris, 1918.

15. Patten BM, Engel WK. Phosphate and parathyroid disorders associated with the syndrome of amyotrophic lateral sclerosis. In Rowland LP (ed): Human Motor Neuron Diseases. New York: Raven Press, 1982, p 181

16. Provinciali L, Laurenzi NA, Vesprini L, Giovagnoli AR, Bartocci C, Montroni M, Bagnarelli P, Clementi M, Veraldo PE. Immunity assessment in the early stages of amyotrophic lateral sclerosis: a study of a virus antibodies and lymphocyte subsets. Acta Neurol Scand 1988, 78:449.

17. Reed DM, Kurland LT. Muscle fasciculations in a healthy population. Arch Neurol 1963, 9:363.

18. Rowland LP. Diverse forms of motor neuron diseases. In Rowland LP (ed) : Human Motor Neuron Diseases. New York: Raven Press, 1982, p 1.

19. Skacel M. Esclerose lateral amiotrófica: revisão sobre os aspectos etiopatogênicos e tentativa de reprodução experimental. Tese apresentada para a obtenção do grau de Mestre em Neurologia. Universidade Federal do Rio de Janeiro. Rio de Janeiro, 1989.

20. Spencer PS, Schaumburg HH. The pathogenesis of motor neuron disease: perspectives from neurotoxicology. In Rowland LP (ed): Human Motor Neuron Diseases. New York: Raven Press, 1982, p 249.

21. Tandau R, Bradley WG. Amyotrophic lateral sclerosis, Part 2: Etiopathogenesis. Ann



